\title{
Molecular comparison of pathogenic bacteria from pear trees in Japan and the fire blight pathogen Erwinia amylovora
}

\author{
Won-Sik Kim, Maja Hildebrand, Susanne Jock and Klaus Geider
}

Max-Planck-Institut für Zellbiologie, Rosenhof, 68526 Ladenburg, Germany
Author for correspondence: Klaus Geider. Tel: +496203106 117. Fax: +496203106122. e-mail: kgeider@zellbio.mpg.de

\begin{abstract}
Several strains of the genus Erwinia, which were isolated in Japan from pear trees with necrotic symptoms that resembled fire blight, and tentatively identified as Erwinia amylovora, were reinvestigated for their relationship to the fire blight pathogen. These isolates produced ooze on slices of immature pears and were mucoid on $\mathrm{MM2Cu}$ agar plates, but did not synthesize levan and did not give the expected PCR signals with several primer pairs specific for Erwinia amylovora. The isolates tested positive with PCR primers designed to detect the novel pear pathogen Erwinia pyrifoliae, which was isolated from Nashi pear trees in South Korea. The nucleotide sequence analysis of a DNA fragment preceding the gene cluster for exopolysaccharide synthesis revealed a closer relationship to Erwinia pyrifoliae than to Erwinia amylovora. Plasmid profiles, protein patterns and genomic DNA analysed by PFGE after Xbal and Spel digestion were different than Erwinia amylovora. Experiments with strains of Erwinia amylovora isolated from raspberry (Rubus sp.), Erwinia mallotivora and Enterobacter pyrinus also did not reveal a relationship between these bacteria and the Japanese Erwinia strains. The latter are not identical to Erwinia pyrifoliae, but possess many similar features to this pathogen that causes Asian pear blight. It is concluded that pathogenic bacteria isolated in Japan from pear trees with symptoms resembling fire blight are possibly different from Erwinia amylovora.
\end{abstract}

Keywords: Asian pear blight, Erwinia pyrifoliae, nucleotide sequence alignment, PCR signals, PFGE

\section{INTRODUCTION}

Several years ago, shoot blight symptoms were observed on Asian pears (Pyrus pyrifolia) in orchards on the Japanese island of Hokkaido. The pathogen was tentatively designated in Japan as Erwinia amylovora pv. pyri (Goto, 1992). In two subsequent reports, isolates from necrotic tissues of Japanese Nashi pear trees were further investigated for their relationship to Erwinia amylovora (Beer et al., 1996; Kim et al., 1996). From microbiological tests, BIOLOG assays and DNA hybridization studies, a close resemblance with Erwinia

Abbreviations: EPS, exopolysaccharide; ITS, intergenic transcribed spacer; SSR, short sequence DNA repeat.

The EMBL accession numbers for the sequences reported in this paper are AJ311829 (Ej546), AJ311830 (Ej617), AJ311831 (Ej557), AJ311832 (Ej562), AJ311833 (Ej556), AJ311834 (Ej546a), AJ311835 (Ep1/96), AJ311836 (Ea1/79) and AJ311837 (IL6). amylovora was suggested, confirming conclusions of a Japanese report from 1981 (cited in Beer et al., 1996) about the occurrence of fire blight in Japan.

Recently, a bacterial pathogen, Erwinia pyrifoliae, was characterized as a novel Erwinia species that also causes necrotic symptoms on Asian pears (Kim et al., 1999; Rhim et al., 1999). The Asian pear pathogen was first detected in orchards near Chuncheon, South Korea, and has not been reported to occur in other countries. In several respects, this pathogen resembled Erwinia amylovora, the causative agent of fire blight of apple (Malus domestica L.) and European pear (Pyrus communis). The nucleotide sequence of the $16 \mathrm{~S}$ rDNA from Erwinia pyrifoliae was almost identical with the corresponding sequence of Erwinia amylovora, and Erwinia pyrifoliae also produced ooze on slices of immature European and Asian pears. Biotype 100 and BIOLOG profiles, DNA-DNA hybridization kinetics, 
as well as nucleotide sequence analysis of the intergenic transcribed spacer region between the $16 \mathrm{~S}$ and $23 \mathrm{~S}$ rDNA distinguished Erwinia pyrifoliae from Erwinia amylovora. Based on these features it was classified as a new species within the genus Erwinia (Kim et al., 1999). Additionally, Erwinia pyrifoliae does not contain a plasmid identical to pEA29 of Erwinia amylovora (Rhim et al., 1999) and its PFGE pattern after an XbaI digest of genomic DNA is distinct from Erwinia amylovora ( $\mathrm{S}$. Jock \& K. Geider, unpublished). Other bacteria connected to necrotic symptoms of Asian pears include Enterobacter pyrinus in Korea (Chung et al., 1993) and Erwinia mallotivora in Japan (Goto, 1992).

The claim of the existence of fire blight in Japan caused by Erwinia amylovora was reinvestigated in this study. Isolates from Japanese orchards described by Beer $e t$ al. (1996) and Kim et al. (1996) were characterized for molecular properties and were found to differ from Erwinia amylovora, but to be related to Erwinia pyrifoliae, the novel species causing Asian pear blight (Kim et al., 1999; Rhim et al., 1999).

\section{METHODS}

Bacterial strains and plasmids. Five Erwinia cultures, which originated from Nashi pear trees in Japanese orchards with symptoms resembling fire blight, were obtained from S. Beer,
Cornell University, Ithaca, NY, USA. The strains were previously classified as Erwinia amylovora (Beer et al., 1996; Kim et al., 1996). Two colony types were observed on LB agar from culture 'Ea546'; a small and a large colony were separately cultured and labelled Ejp546 and Ejp546a, respectively. All the strains used for comparison are listed in Table 1. Plasmids used in cloning experiments and molecular details of Erwinia amylovora strain Ea1/79, Erwinia pyrifoliae strain Ep1/96 and Enterobacter pyrinus 90.2 were described by Kim et al. (1999).

Microbiological characterization of strains. All strains were first assayed on semi-selective medium (Bereswill et al., 1998). $\mathrm{MM} 2 \mathrm{Cu}$ is a minimal medium with a high content of asparagine and causes the formation of yellow, mucoid colonies for Erwinia amylovora. MM1Cu with a low concentration of asparagine prevents growth of Erwinia amylovora. LBsuc is Luria-Bertani broth with $5 \%$ sucrose, used to determine levan production. The oxidative/ fermentative reaction was determined according to Hugh \& Leifson (1953).

PCR assays for identification of Erwinia strains from Japan. Primers for detection and identification of Erwinia amylovora as well as Erwinia pyrifoliae are listed in Table 2 and their target positions are outlined in Fig. 1. The oligonucleotides were synthesized with a Beckman Oligo 1000M DNA Synthesizer. The PCR reactions were done in $50 \mu \mathrm{l}$ with dilutions of bacterial cell cultures and $0.5 \mathrm{U}$ Tth polymerase (Amersham Pharmacia) or $2 \mathrm{U}$ Taq polymerase as described by Bereswill et al. $(1992,1995)$ for all primer pairs, and 30

Table 1. Erwinia strains isolated from necrotic tissue of Asian pear trees in Japan and other bacterial strains used for comparison

Cultures of the Japanese strains (a) were provided by Dr S. Beer, Cornell University, Ithaca, NY, USA. ", **, derived from a small and a large colony of culture Ea546, respectively. Ejp546a may not be an independent isolate. CFBP, Collection Française des Bactéries Phytopathogènes (French Collection of Phytopathogenic Bacteria); KCTC, Korean Collection of Type Cultures.

(a) Erwinia strains from Japan

\begin{tabular}{|c|c|c|c|c|c|c|}
\hline New name & Previous name & Other name & Year of isolation & Host & Location & Source \\
\hline Ejp546* & Ea546 & EA7971 & 1979 & Asian pear & Hokkaido & A. Tanii \\
\hline Ejp546a** & Ea546 & EA7971 & 1979 & Asian pear & Hokkaido & A. Tanii \\
\hline Ejp556 & Ea556 & TP9405 & 1994 & Asian pear & Hokkaido & A. Tanii \\
\hline Ejp557 & Ea557 & EA9471 & 1994 & Asian pear & Hokkaido & A. Tanii \\
\hline Ejp562 & Ea562 & EA9476 & 1994 & Asian pear & Hokkaido & A. Tanii \\
\hline Ejp617 & Ea617 & 144 & 1996 & Asian pear & Japan & R. Roberts \\
\hline
\end{tabular}

(b) Other strains

\begin{tabular}{|lll|}
\hline Strain & \multicolumn{1}{c|}{ Origin } & \multicolumn{1}{c|}{ Reference } \\
\hline Ea1/79 & $\begin{array}{c}\text { Erwinia amylovora, from Cotoneaster sp., Germany, 1979, applied as } \\
\text { a strain from fruit trees/ornamentals }\end{array}$ & Falkenstein et al. (1988) \\
IL6 & Erwinia amylovora, from raspberry (Rubus sp.), USA & Kim \& Geider (1999) \\
Ep1/96 & Erwinia pyrifoliae, from Nashi pear, Korea, 1996 & Kim et al. (1999) \\
$90.2^{\mathrm{T}}$ & Enterobacter pyrinus, from Nashi pear, Korea, KCTC 2520 & KCTC \\
CFBP2503 & Erwinia mallotivora, ATCC 29573 & CFBP \\
DC283 & Pantoea stewartii (formerly Erwinia stewartii), USA & Bereswill et al. (1998) \\
DH5 $\alpha$ & Mutated Escherichia coli K-12 & BRL \\
\hline
\end{tabular}


Table 2. Primers used for differentiation of Erwinia spp.

Positions of the primers can be deduced from Fig. 1. The expected sizes of the products are $(\mathrm{kb}): \mathrm{fd} 2 / \mathrm{rP} 1,1 \cdot 4 ; \mathrm{EP} 16 \mathrm{~A} / \mathrm{EPIG} 2 \mathrm{c}, 0 \cdot 70$; P29A/P29B, 1·0; RS1/RS2c, 0·15; AMS1/AMS2c, 1·2; AMS3/AMS4c, 0·83; AMSbL/AMSbR, 1·6; CPS1/CPS2c, $1 \cdot 2$.

\begin{tabular}{|c|c|c|}
\hline Primer & Sequence $\left(5^{\prime}-3^{\prime}\right) /$ features & Reference/source \\
\hline \multicolumn{3}{|c|}{ From rRNA region: } \\
\hline $\mathrm{fd} 2 *$ & AGAGTTTGATCATGGCTCAG/16S rDNA consensus primer & Weisburg et al. (1991) \\
\hline rP1* & ACGGTTACCTTGTTACGACTT/16S rDNA consensus primer & Weisburg et al. (1991) \\
\hline EP16A & AGATGCGGAAGTGCTTCG/from 16S rDNA of Erwinia pyrifoliae & Kim et al. (2001) \\
\hline EPIG2c & ACCGTTAAGGTGGAATC/from ITS of Erwinia pyrifoliae & Kim et al. (2001) \\
\hline \multicolumn{3}{|c|}{ From plasmid pEA29: } \\
\hline P29A† & CGGTTTTTAACGCTGGG/from Erwinia amylovora plasmid pEA29 & Bereswill et al. (1992) \\
\hline $\mathrm{P} 29 \mathrm{~B}+$ & GGGCAAATACTCGGATT/from Erwinia amylovora plasmid pEA29 & Bereswill et al. (1992) \\
\hline RS1 & ACCTCAGTGCGATTACAG/used to analyse SSRs in pEA29 & Kim \& Geider (1999) \\
\hline RS2c & GTCCCATTCTGTGTAAAG/used to analyse SSRs in pEA29 & Kim \& Geider (1999) \\
\hline \multicolumn{3}{|c|}{$\begin{array}{l}\text { From region for EPS } \\
\text { synthesis: }\end{array}$} \\
\hline AMS1 & $\begin{array}{l}\text { GGCAGAAGTTGTGAGCA/from ams of Erwinia amylovora } \\
\text { corresponding to position of CPS1 in Erwinia pyrifoliae }\end{array}$ & Kim et al. (2001) \\
\hline AMS2c $\neq$ & $\begin{array}{l}\text { AAACAGGTGCGCCGAATA/from ams of Erwinia amylovora } \\
\text { corresponding to position of CPS2c in Erwinia pyrifoliae }\end{array}$ & Kim et al. (2001) \\
\hline AMS3 & GACGGATCGGCAATCCATTC/from region preceding amsG & This work \\
\hline AMS4c & CGCGCATAATTAGCTCT/from region preceding ams $G$ & This work \\
\hline AMSbL & GCTACCAGCAGGGTGAG/from ams region of Erwinia amylovora & Bereswill et al. (1995) \\
\hline AMSbR & TCATCACGATGGTGTAG/from ams region of Erwinia amylovora & Bereswill et al. (1995) \\
\hline CPS1S & CGCGGAAGTGGTGAGAA/cps region of Erwinia pyrifoliae & Kim et al. (2001) \\
\hline $\mathrm{CPS} 2 \mathrm{c} \mathbb{S}$ & GAACAGATGTGCCGAGTA/cps region of Erwinia pyrifoliae & Kim et al. (2001) \\
\hline
\end{tabular}

*Expected fragments after a digest of the $1 \cdot 4 \mathrm{~kb}$ PCR fragment from Erwinia amylovora with HaeIII: 0·32, 0·22, 0·20, 0·17, 0·16, 0·12 kb. Expected fragments with Sau3A: 0.6, 0.42, 0.26, 0.15 kb.

†Expected fragments after a digest of the $1 \cdot 0 \mathrm{~kb}$ PCR fragment from Erwinia amylovora strain Ea1/79 with Sau3A: 0.26, 0.17, 0.17, $0 \cdot 11,0 \cdot 11,0 \cdot 1,0 \cdot 05$ and $0 \cdot 04$ bp.

$\ddagger$ Expected fragments after a digest of the $1 \cdot 2 \mathrm{~kb}$ PCR fragment from Erwinia amylovora with HaeIII: 0·57, 0·21, 0·14, 0·13, 0·09, 0.08 kb. \Expected fragments after a digest of the $1 \cdot 2 \mathrm{~kb}$ PCR fragment from Erwinia pyrifoliae with HaeIII: 0.65, 0·27, 0·21, 0.09 kb.

cycles at $94{ }^{\circ} \mathrm{C}$ for $15 \mathrm{~s}, 52{ }^{\circ} \mathrm{C}$ for $15 \mathrm{~s}$ and $72{ }^{\circ} \mathrm{C}$ for $30 \mathrm{~s}$ were used for denaturation, annealing and polymerization, respectively. The expected product sizes are given in Table 2.

Cloning and sequencing of DNA fragments from the 16S rRNA nucleotide sequence and a region preceding the gene cluster for exopolysaccharide (EPS) synthesis. For cloning of the $16 \mathrm{~S}$ rDNA, primers $\mathrm{fd} 2$ and $\mathrm{rP} 1$ were mixed with 0.5 units Tth DNA polymerase (Pharmacia) and amplified as described by Kim et al. (1999) with approximately 10000 cells and $0 \cdot 2 \mathrm{mM}$ dATP, dCTP, dGTP and dTTP (Roche). Thirty cycles of amplification were carried out in an Eppendorf Mastercycler 5330. Denaturation was done at $94{ }^{\circ} \mathrm{C}$ for $45 \mathrm{~s} \mathrm{in}$ the first cycle and for $30 \mathrm{~s}$ in subsequent cycles, followed by $45 \mathrm{~s}$ at $52{ }^{\circ} \mathrm{C}$ for annealing and $30 \mathrm{~s}$ at $72{ }^{\circ} \mathrm{C}$ for polymerization. The $1.4 \mathrm{~kb}$ DNA product was eluted from the gel, purified with a PCR purification kit (Qiagen) and the product inserted into pGEM-T (Promega). A DNA fragment upstream the gene cluster for EPS synthesis was amplified with primers AMS3 and AMS4c and inserted into plasmid pGEMT. Nucleotide sequencing of the cloned fragments was performed with an automatic sequencer (ALFexpress; Amersham Pharmacia Biotech). The DNA sequences were analysed with the programs Clone Manager v. 5 and Align v. 4 (Scientific \& Educational Software, State Line, PA, USA). The Align program was also used for the dendrogram constructed with a distance-based tree building method using the neighbour-joining algorithm.

The Erwinia amylovora sequence overlaps with nt 234-1064 in the nucleotide sequence deposited in the EMBL sequence database under accession no. X77921 for strain CFBP1430.

SDS-PAGE analysis. Proteins from cell extracts were separated by PAGE using a $5 \%$ stacking gel and a $10 \%$ resolving gel (Sambrook et al., 1989). Whole-cell lysates were prepared for electrophoresis from $1.5 \mathrm{ml}$ cultures, all grown overnight in LB medium. The cell pellets were resuspended in $100 \mu$ l SDSPAGE sample buffer with $\beta$-mercaptoethanol and boiled for $5 \mathrm{~min}$. Ten microlitres of supernatant was applied and the protein bands were stained with Coomassie blue R-250. Fructose-6-phosphate kinase $(85 \mathrm{kDa})$, bovine serum albumin $(67 \mathrm{kDa})$, pyruvate kinase $(66 \mathrm{kDa})$ and carbonic anhydrase $(30 \mathrm{kDa})$ were used as size markers.

Plasmids and PFGE analyses. Total plasmid DNA was isolated after alkaline lysis and purified with a Nucleobond AX column 
(a)

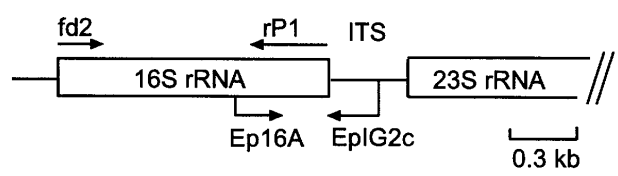

(b)

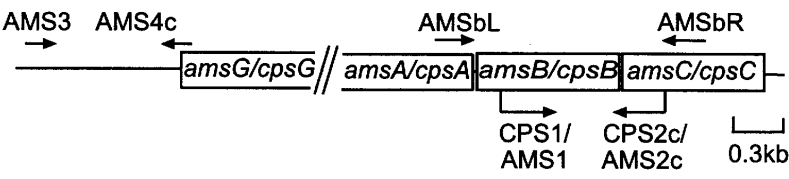

(c)

\begin{tabular}{|c|c|c|}
\hline$\stackrel{\text { RS1 }}{\longrightarrow}$ & RS2c & $0.1 \mathrm{~kb}$ \\
\hline & & 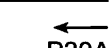 \\
\hline
\end{tabular}

Fig. 1. A schematic presentation of primers used for identification of the Erwinia strains from Japan. The nucleotide sequences designed for the detection of Erwinia amylovora and Erwinia pyrifoliae are listed in Table 2. (a) ITS region with parts of $16 \mathrm{~S}$ and $23 \mathrm{~S}$ rRNA; (b) region with EPS synthesis genes of Erwinia amylovora (ams) and the corresponding region of Erwinia pyrifoliae (cps); (c) a DNA fragment from pEA29 of Erwinia amylovora corresponding to fragment $F$ of pEA29-Ea88 in the map of McGhee \& Jones (2000) applied to detection of the pathogen (Bereswill et al., 1992) and for SSR analysis (Kim \& Geider, 1999).

(Macherey and Nagel) according to the manufacturer's instructions. Preparation of the plugs for PFGE, digestion with restriction enzymes and separation of the DNA fragments were as described by Zhang \& Geider (1997) and Zhang et al. (1998).

Plant assays. Virulence tests with slices of immature pears and with apple seedlings, as well as hypersensitive response assays, were done as described by Rhim et al. (1999). Infiltration of three tobacco leaves (cultivar 'Samsun') was done with bacterial suspensions in water at $10^{8}$ c.f.u. $\mathrm{ml}^{-1}$. For the other inoculations, bacterial cultures were grown for $1 \mathrm{~d}$ in nutrient broth and cell suspensions transferred to several pear slices or wounded leaves of pear seedlings with a toothpick. Negative controls were done with an Escherichia coli strain.

\section{RESULTS}

\section{Properties of the Japanese Erwinia strains in assays on agar}

On MM2Cu agar plates (Bereswill et al., 1998), the Erwinia strains from Japan formed white to pale-yellow mucoid colonies, typical for Erwinia pyrifoliae (Rhim et al., 1999), in contrast to Erwinia amylovora colonies on MM2Cu agar (Bereswill et al., 1998) which display an intermediate to bright-yellow colour. Erwinia mallotivora did not grow on $\mathrm{MM} 2 \mathrm{Cu}$ agar. Neither the Japanese Erwinia strains nor the Erwinia pyrifoliae strains produced levan on LB-sucrose, whereas Erwinia amylovora did. As found for Erwinia amylovora and Erwinia pyrifoliae strains, the Japanese isolates did not (a)

(b)

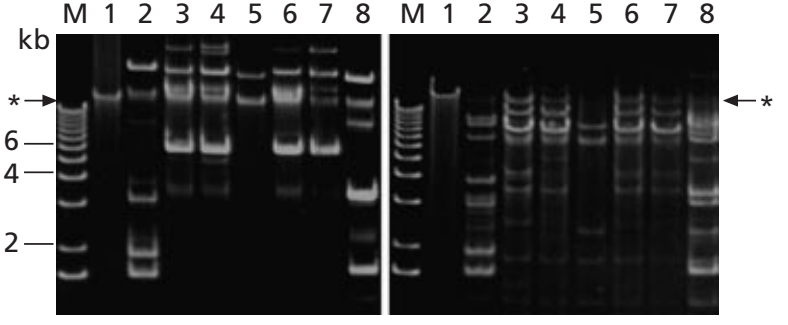

Fig. 2. Plasmid profiles of Erwinia strains from Japan, Erwinia amylovora and Erwinia pyrifoliae. (a) Undigested; (b) BamHIdigested. Lanes: 1, Erwinia amylovora Ea1/79; 2, Erwinia pyrifoliae Ep1/96; 3, Ejp546a; 4, Ejp546; 5, Ejp556; 6, Ejp557; 7, Ejp562; 8, Ejp617; *, position of plasmid pEA29; M, marker DNA with $1 \mathrm{~kb}$ ladder (BRL). The agarose gel was run at $100 \mathrm{~V}$ for $90 \mathrm{~min}$.

grow on MM1Cu agar (Bereswill et al., 1998). They did not fluoresce on King's medium B and were facultatively anaerobic in Hugh-Leifson (Hugh \& Leifson, 1953) agar, indicating that the isolates were not pseudomonads.

\section{Plant assays for confirmation as plant pathogens}

The Japanese Erwinia strains produced a hypersensitive response on tobacco similar to Erwinia amylovora and Erwinia pyrifoliae. As with Erwinia pyrifoliae, they were not pathogenic on apple seedlings (cv. 'Golden Delicious'), but produced ooze on slices of immature pears as do strains of Erwinia amylovora and Erwinia pyrifoliae (Rhim et al., 1999). In contrast, no ooze was produced after inoculation of pear slices with Erwinia mallotivora or Enterobacter pyrinus.

\section{Differentiation by plasmid profiles and protein patterns}

Plasmids from the Japanese Erwinia strains, Erwinia amylovora and Erwinia pyrifoliae were separated on $1 \%$ agarose gels (Fig. 2). At least one plasmid of the Erwinia strains from Japan was significantly larger than $29 \mathrm{~kb}$, the size of plasmid pEA29, commonly found in Erwinia amylovora, and in all strains but Ejp556 at least one smaller plasmid was observed. Plasmid pEA29 (Fig. $2 \mathrm{a}$, lane 1 ) carries a single Bam HI site (Falkenstein et al., 1988) and was linearized after digestion (Fig. 2b, lane 1). Digestion with BamHI cleaved most of the other large plasmids (Fig. 2a) into several DNA fragments (Fig. 2b). The plasmid patterns of four strains, Ejp546, Ejp546a, Ejp557 and Ejp562, were similar to each other, but Ejp556 did not carry the plasmid migrating as a supercoil at the position of a $6 \mathrm{~kb}$ DNA fragment. Ejp617 produced different plasmid profiles than the other strains and resembled that of Erwinia pyrifoliae Ep1/96 except that it contained only one low-molecular-mass plasmid migrating at the position of a $2 \mathrm{~kb}$ DNA fragment or below. These small plasmids were not cleaved by 


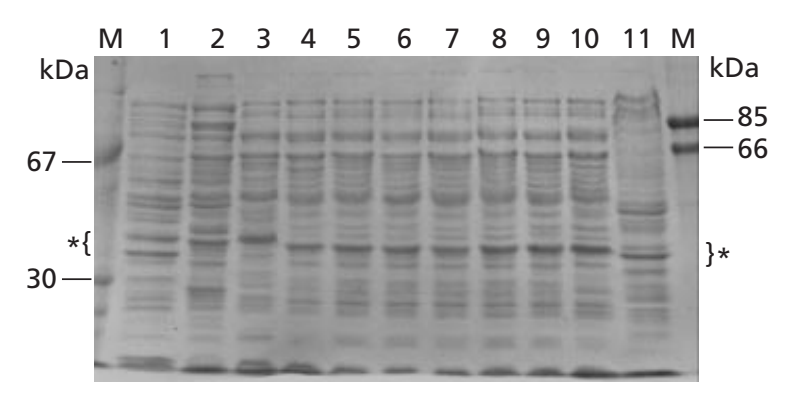

Fig. 3. Comparison of total protein patterns from cell lysates. Ten microlitre aliquots from $100 \mu$ l cell lysate were separated by $10 \%$ SDS-PAGE and stained with Coomassie blue. Lanes: 1, Escherichia coli $\mathrm{DH} 5 \alpha$; 2, Pantoea stewartii DC283; 3, Erwinia amylovora Ea1/79; 4, Ejp546; 5, Ejp546a; 6, Ejp556; 7, Ejp557; 8, Ejp562; 9, Ejp617; 10, Erwinia pyrifoliae Ep1/96; 11, Enterobacter pyrinus; M, molecular mass markers; *, position of proteins different to Erwinia amylovora.

BamHI, similar to the small plasmids of Erwinia pyrifoliae (Rhim et al., 1999). The complex plasmid pattern for the Erwinia strains from Japan supported the notion that they were not Erwinia amylovora.

Cells of the Erwinia strains from Japan and other bacteria were lysed and their protein patterns determined on an SDS-polyacrylamide gel (Fig. 3). All Japanese Erwinia strains gave essentially the same protein pattern, which was distinct from Escherichia coli, Pantoea stewartii and Enterobacter pyrinus, which is another micro-organism from necrotic Nashi pear trees in Korea (Chung et al., 1993). The protein pattern of Erwinia pyrifoliae strain Ep1/96 was similar to that of the Japanese strains. Only a few differences in lowmolecular-mass proteins were observed. Similarity coefficient values (Nei \& Li, 1979) of six Erwinia strains from Japan and Escherichia coli, Pantoea stewartii, Erwinia amylovora and Erwinia pyrifoliae were 0.55, $0.58,0.67$ and 0.95 , respectively. This result confirmed a closer relationship of the Japanese Erwinia strains to Erwinia pyrifoliae than to Erwinia amylovora.

\section{PFGE patterns of Spel and Xbal fragments}

The genomes of the Japanese Erwinia strains Ejp546, Ejp546a, Ejp556, Ejp557, Ejp617 and Ejp562 were digested with restriction enzyme XbaI and the DNA fragments were analysed by PFGE. The band patterns were partially related to each other, but not to those of Erwinia amylovora (Fig. 4), which is highly homogeneous in its PFGE patterns after an $\mathrm{XbaI}$ digest (Zhang \& Geider, 1997; Zhang et al., 1998). The PFGE patterns of the Japanese strains were also different from Erwinia pyrifoliae Ep1/96, which shares the XbaI pattern with several other Erwinia pyrifoliae strains (unpublished). Similarities of XbaI DNA fragments were found for strains Ejp546a, Ejp557 and Ejp562 (Fig. 4). The XbaI DNA fragments of strains Ejp546 and Ejp546a differed from each other. Different pattern types were also observed for strains Ejp556 and Ejp617. Due to the complex patterns and overlapping bands in individual

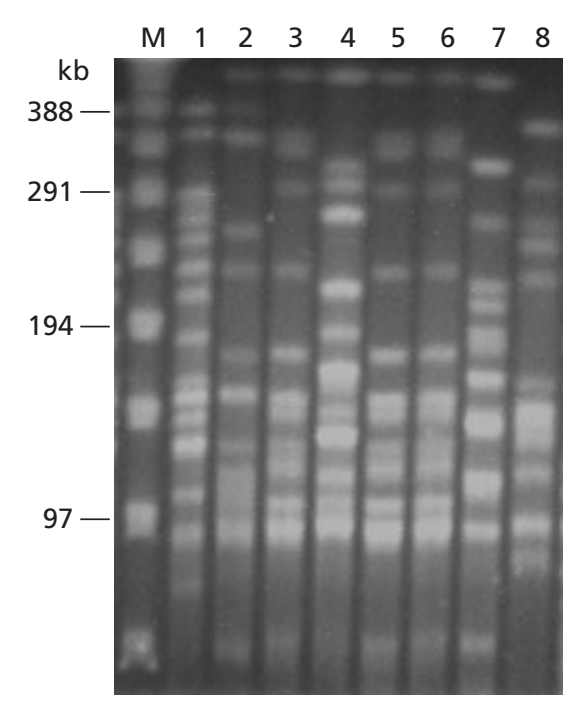

Fig. 4. PFGE pattern of the Japanese Erwinia strains, Erwinia amylovora and Erwinia pyrifoliae after an Xbal digest. Lanes: $M$, size marker of $\lambda$ DNA multimers; 1 , Ea1/79; 2 , Ejp546; 3 , Ejp546a; 4, Ejp556; 5, Ejp557; 6, Ejp562; 7, Ejp617; 8, Ep1/96.

profiles, similarity coefficient values (Nei \& Li, 1979) could only be roughly estimated for a close relationship of Ejp546a, Ejp557 and Ejp562, a less close relationship of Ejp546, Ejp617 and Ejp556, and even less with Erwinia pyrifoliae and Erwinia amylovora. PFGE analysis after an SpeI digest gave matching patterns for Ejp546a, Ejp557 and Ejp562, whereas Ejp546 differed from these strains in two bands (data not shown). As for XbaI digests, strains Ejp556 and Ejp617 were also divergent in their SpeI fragments. No strong relationship in their patterns was found with Erwinia pyrifoliae or Erwinia amylovora.

\section{PCR analysis with primers from plasmid pEA29 and from EPS- and rDNA-encoding regions}

To further confirm differences between the Japanese Erwinia strains and Erwinia amylovora, primers P29A and P29B, which were derived from plasmid pEA29 and have been used for specific detection of the fire blight pathogen, were applied. They also produced a PCR product of $1 \mathrm{~kb}$ with Erwinia amylovora strain IL6, isolated from Rubus sp. As reported by Beer et al. (1996) for the Erwinia strains from Japan, the plasmid primers derived from pEA29 produced a band slightly larger than the band specific for detection of Erwinia amylovora. A subsequent Sau3A digest of the amplified DNA produced bands from the Erwinia amylovora plasmid of the expected sizes (Table 3). Sau3A digested the $1.2 \mathrm{~kb}$ band, amplified from chromosomal or plasmid DNA of the Japanese Erwinia strains with primers $\mathrm{P} 29 \mathrm{~A}$ and $\mathrm{P} 29 \mathrm{~B}$, into fragments of $0 \cdot 60,0 \cdot 17$, $0 \cdot 17,0 \cdot 11,0 \cdot 05$ and $0.035 \mathrm{~kb}$. In the case of strain Ejp546, the large DNA fragment of the digest was slightly smaller than the corresponding fragment of the other strains. Comparison of the two sets indicated different PCR products for the Erwinia strains from Japan and 
Table 3. Summary and comparison of molecular properties of the Erwinia strains from Japan, Erwinia pyrifoliae strain Ep1/96 and Erwinia amylovora strain Ea1/79

The standard strain used for Erwinia amylovora was Ea1/79 with the PFGE pattern (XbaI) of the type strain S59/5, and the standard for Erwinia pyrifoliae was Ep1/96 with the PFGE pattern (SpeI) of the type strain Ep16/96. The data for Ea1/79 and Ep1/96 are representative for many strains of the species. $\mathrm{db}$, Double band; tb, triple band.

\begin{tabular}{|c|c|c|c|}
\hline Molecular property & $\begin{array}{c}\text { Erwinia strains from } \\
\text { Japan }\end{array}$ & Ep1/96 & Ea1/79 \\
\hline \multicolumn{4}{|l|}{ Plasmids and PCR products: } \\
\hline \multicolumn{4}{|l|}{ Plasmid preparations: } \\
\hline Undigested & Several & Several & One (pEA29) \\
\hline BamHI digest & Multiple cuts & Multiple cuts & Single cut \\
\hline \multicolumn{4}{|l|}{ PCR signal with primers: } \\
\hline P29A,B & $+(1 \cdot 2 \mathrm{~kb})$ & Weak bands & $+(1 \cdot 0 \mathrm{~kb})$ \\
\hline Sau3A digest of band & $\begin{array}{l}0 \cdot 60,0 \cdot 17(\mathrm{db}), 0 \cdot 11 \\
0 \cdot 05,0 \cdot 035 \mathrm{~kb}\end{array}$ & & $\begin{array}{l}0 \cdot 26,0 \cdot 17(\mathrm{db}), 0 \cdot 11(\mathrm{tb}) \\
0 \cdot 05,0 \cdot 035 \mathrm{~kb}\end{array}$ \\
\hline RS1, RS2 & No signal & No signal & $+(\sim 0 \cdot 15 \mathrm{~kb})$ \\
\hline \multicolumn{4}{|c|}{ PCR products from ams/cps regions: } \\
\hline CPS1, CPS2c (Ep) & $+(1 \cdot 2 \mathrm{~kb})$ & $+(1 \cdot 2 \mathrm{~kb})$ & No signal \\
\hline HaeIII digest of band $(\mathrm{kb})$ & $0 \cdot 65,0 \cdot 27,0 \cdot 21,0.09$ & $0 \cdot 65,0 \cdot 27,0 \cdot 21,0 \cdot 09$ & - \\
\hline AMSbL, AMSbR & No signal & No signal & $1.6 \mathrm{~kb}$ \\
\hline AMS1, AMS2c & No signal & No signal & $1 \cdot 2 \mathrm{~kb}$ \\
\hline \multicolumn{4}{|c|}{$\begin{array}{l}\text { Nucleotide sequences of region in front } \\
\text { of EPS-synthesis-encoding genes: }\end{array}$} \\
\hline Identity to Ejp546 & $99-96 \%$ (for others) & $95 \%$ & $83 \%$ \\
\hline \multicolumn{4}{|l|}{ Protein pattern: } \\
\hline Bands on gel & Similar to Ep1/96 & Reference & Distinct from Ep1/96 \\
\hline
\end{tabular}

Erwinia amylovora. Primers used previously to determine the size of the short sequence DNA repeats (SSR) in plasmid pEA29 (Kim \& Geider, 1999) did not produce a PCR signal with the Erwinia strains from Japan or with Erwinia pyrifoliae (data not shown).

With another primer pair specific for the detection of Erwinia amylovora, derived from the region for amylovoran synthesis (ams) (Bereswill et al., 1995), a product was obtained for Erwinia amylovora from fruit trees/ornamentals and from raspberry (Rubus sp.), but not for the Japanese Erwinia strains or for Erwinia pyrifoliae (data not shown). The cps region of Erwinia pyrifoliae encodes an EPS similar to amylovoran synthesized by proteins homologous to Ams proteins (unpublished). On the other hand, the nucleotide sequence of the cps region is not identical to the ams region of Erwinia amylovora and PCR primers were designed to discriminate Erwinia pyrifoliae from Erwinia amylovora (W.-S. Kim, S. Jock, J.-P. Paulin, S.-L. Rhim \& K. Geider, unpublished). With the Japanese Erwinia strains, these primers amplified a DNA fragment of the same size as for Erwinia pyrifoliae (Fig. 5a). In a HaeIII digest of the PCR band, strains Ejp557 and Ejp617 produced DNA fragments identical to each other and with fragments of Erwinia pyrifoliae strain Ep1/96
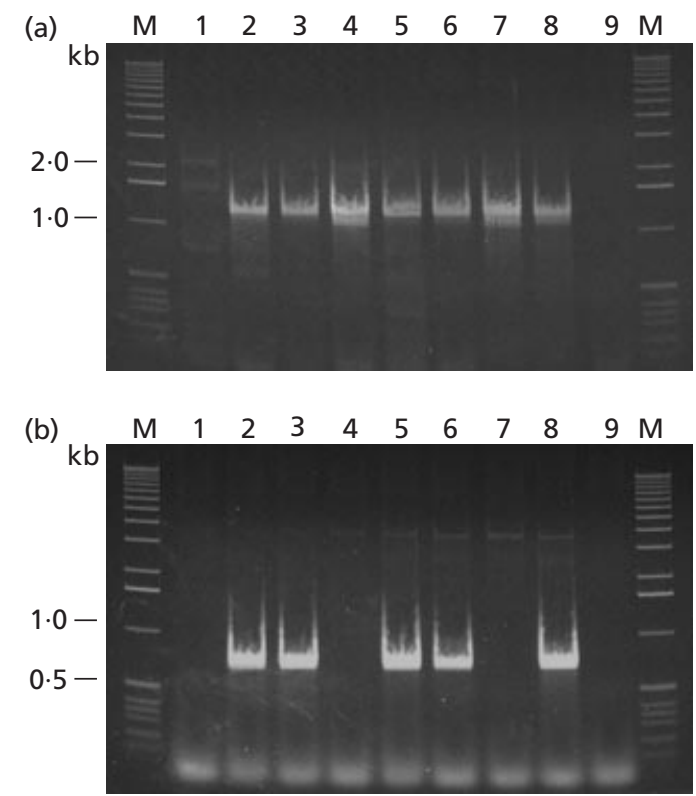

Fig. 5. $P C R$ assay with (a) cps primers (CPS1/CPS2c) and (b) $16 \mathrm{~S}$ ITS primers (EP16A/EPIG2c) from Erwinia pyrifoliae. Lanes: 1, Erwinia amylovora Ea1/79; 2, Ejp546; 3, Ejp546a; 4, Ejp556; 5, Ejp557; 6, Ejp562; 7, Ejp617; 8, Erwinia pyrifoliae Ep1/96; 9, water control; M, 1 kb ladder marker DNA. 
Ep1/96 6 Ejp strains Ea1/79 IL6 gttatggatgcaaagctatgttgacactacttatcaggcataccgcatttcataatt-t

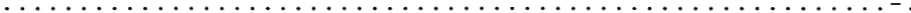
...ccct........t...tg.g...g...aa.a...c.c.a.t....a. ...ccct...-.....t...tg.g...g....aa..t.c..c.a.t....a.
Fig. 6. Alignment of part of the nucleotide sequence preceding the gene clusters for EPS synthesis to compare Erwinia pyrifoliae Ep1/96 and the six Japanese Erwinia strains used in this study with Erwinia amylovora strains Ea1/79 and IL6. (data not shown). When primers from the ams operon of Erwinia amylovora (AMS1 and AMS2c) were applied, these did not produce a signal with the Erwinia strains from Japan nor with Erwinia pyrifoliae. In addition, the HaeIII digestion pattern from the DNA fragments obtained from Erwinia amylovora with primers AMS1 and AMS2c was different from the HaeIII pattern obtained with primers CPS1 and CPS2c from Erwinia pyrifoliae and the Japanese strains (Tables 2, 3). Accordingly, the region encoding EPS synthesis in the Japanese Erwinia strains is closely related to Erwinia pyrifoliae, but less related to Erwinia amylovora.

Another PCR assay for Erwinia pyrifoliae was done with primers EP16A and EPIG2c from the 16S rRNA intergenic transcribed spacer (ITS) region of Erwinia pyrifoliae (Figs $1 \mathrm{a}$ and $5 \mathrm{~b}$ ). When these primers were used to assay the Japanese Erwinia strains Ejp546, Ejp546a, Ejp557 and Ejp652, a DNA fragment of $0.7 \mathrm{~kb}$ was produced, similar to the signal from the Erwinia pyrifoliae strains (eight different strains). The product was not obtained with strains Ejp556 and Ejp617. No signals were obtained for Erwinia amylovora with primers EP16A and EPIG2c or CPS1 and CPS2c.

The 16S rDNA of the Japanese Erwinia strains was amplified with consensus primers (Weisburg et al., 1991) and the PCR product digested with HaeIII. For the Japanese Erwinia strains, Erwinia pyrifoliae and Erwinia amylovora an identical pattern of DNA fragments was found. Sequence analysis of the $16 \mathrm{~S}$ rDNA from the three erwinias revealed $99 \%$ identity (data not shown). This region is apparently highly conserved for the Japanese Erwinia strains, Erwinia pyrifoliae and Erwinia amylovora, and cannot be used for their differentiation.

\section{Sequence analysis of a DNA fragment in front of the chromosomal region encoding EPS synthesis}

Biosynthesis of amylovoran depends on sugar transferases and transport proteins encoded in the ams region of Erwinia amylovora (Bugert \& Geider, 1995). No major ORFs were detected for the nucleotide sequence up to $1 \mathrm{~kb}$ upstream of ams $\mathrm{G}$. Promoter signals can be assumed for the region adjacent to the first gene, ams G. From this region (nt 234-1064 in the sequence deposited in the EMBL nucleotide sequence database under accession no. X77921) 831 bp were subjected to sequence comparison for the Erwinia strains from Japan, Erwinia amylovora strains Ea1/79 (fruit trees/ ornamentals) and IL6 (Rubus sp.), as well as Erwinia

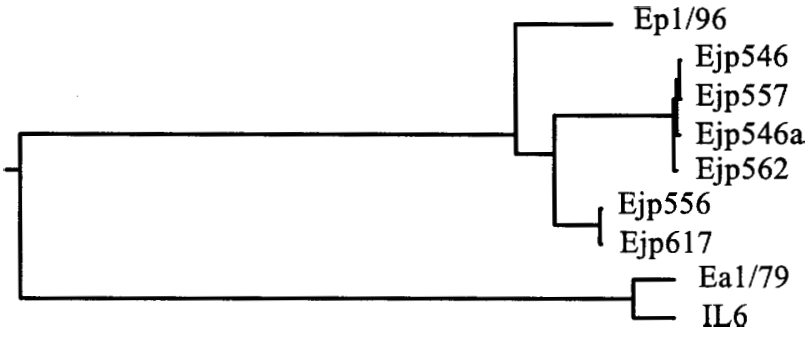

Fig. 7. Dendrogram derived from the nucleotide sequence in front of the EPS-encoding regions for the investigated Erwinia strains from Japan, Ep1/96 (from Nashi pear trees, Korea), and Erwinia amylovora Ea1/79 (fruit tree strain) and IL6 (Rubus strain).

pyrifoliae strain Ep1/96, and a region with significant differences, presented in Fig. 6, was identified. Alignment of the sequence revealed several features for the nine strains investigated: (i) the Erwinia strains from Japan were highly related to each other (96-99\% identity); (ii) Ejp546, Ejp546a, Ejp557 and Ejp562 can be distinguished from Ejp556 and Ejp617 by several mismatches; (iii) Erwinia strains from Japan are more closely related to Erwinia pyrifoliae (95\%) than to Erwinia amylovora strains (fruit tree, $83 \%$; Rubus sp., 84\%); (iv) Erwinia amylovora strains from different hosts (fruit trees/ornamentals or Rubus sp.) carry an almost identical nucleotide sequence in this region. A dendrogram based on these nucleotide sequences placed the Erwinia strains from Japan close to Erwinia pyrifoliae and more distant from Erwinia amylovora (Fig. 7).

\section{DISCUSSION}

Although Erwinia strains from Japan, associated with fire-blight-like symptoms in Japanese pear orchards, have been reported to be Erwinia amylovora (Beer et al., 1996; Kim et al., 1996), a series of molecular data contradicts this claim. Recently, the novel species Erwinia pyrifoliae was described (Kim et al., 1999), which causes necrotic symptoms on shoots of Asian pear trees in Korea (Rhim et al., 1999). Strains of Erwinia sp. from necrotic tissue of Nashi pears, isolated in Japan, were reported to be only weakly virulent on apple seedlings (Beer et al., 1996). This property was confirmed for the strains investigated in this report. On semi-selective plates, by symptoms on immature pears and with molecular tools, these strains were not found to be related to Enterobacter pyrinus, also isolated from 
Nashi pear fruit trees (Chung et al., 1993) or to Erwinia mallotivora, which causes necrotic leaf spots on Nashi pear (Goto, 1992). The nucleotide sequences of the $16 \mathrm{~S}$ rDNA were almost identical for the Japanese pear pathogen, Erwinia pyrifoliae and Erwinia amylovora (Table 3). When the ITS region, which is often indicative for species (Kim et al., 1999), was amplified, multiple DNA fragments were obtained, differing in size as well as the type of the embedded tRNA gene and could therefore not be used for classification of the three erwinias (M. Hildebrand, W.-S. Kim \& K. Geider, unpublished).

On the other hand, several properties, such as the lack of a PCR product with primer pairs of the ams region and primers RS1 and RS2 from pEA29 (Table 3), allowed differentiation of strains Ejp546, Ejp546a, Ejp557, Ejp562, Ejp556 and Ejp617 from Erwinia amylovora. With the Erwinia strains from Japan, the plasmid primers $\mathrm{P} 29 \mathrm{~A}$ and $\mathrm{P} 29 \mathrm{~B}$ gave rise to a DNA fragment around $1 \mathrm{~kb}$ (Beer et al., 1996). Digestion with a restriction enzyme showed differences in the cleavage products in comparison to Erwinia amylovora (Table 3), confirming that the signal with primers P29A and P29B was derived from a sequence not identical to the $1 \mathrm{~kb}$ fragment of pEA29 amplified from Erwinia amylovora. In contrast, the Japanese erwinias resembled Erwinia pyrifoliae in several molecular properties (Table 3). All Japanese Erwinia strains gave a positive PCR signal with the cps primers from Erwinia pyrifoliae and produced identical DNA fragments after a restriction enzyme digest, confirming that the genes for EPS synthesis of the Japanese erwinias are related to the $c p s$ genes of Erwinia pyrifoliae. When the corresponding chromosomal regions preceding the gene clusters for EPS synthesis of Erwinia pyrifoliae, the Erwinia strains from Japan and Erwinia amylovora strains isolated from fruit trees/ornamentals and from Rubus sp. were aligned, the Japanese strains had a closer relationship to Erwinia pyrifoliae than to Erwinia amylovora. The similarity to Erwinia pyrifoliae was confirmed by PAGE of total cellular proteins and by the plasmid profiles. Plasmid pEA29 was found in all Erwinia amylovora strains investigated by DNA hybridization and by PCR assays with plasmid-derived primers (Falkenstein et al., 1988; Bereswill et al., 1992). In contrast to Erwinia amylovora, Erwinia pyrifoliae strains (Rhim et al., 1999) and the Erwinia strains from Japan displayed a complex plasmid pattern. The Rubus sp. strains share a simple plasmid pattern with the Erwinia amylovora strains from fruit trees and ornamentals, and the pEA29 derivatives are largely identical for strains from different hosts (McGhee \& Jones, 2000). Cross-hybridization was observed with labelled DNA from plasmid pEA29 and DNA from Erwinia amylovora fruit tree and Rubus sp. strains, Erwinia pyrifoliae and the Erwinia strains from Japan (S. Jock \& K. Geider, unpublished), limiting the use of total plasmid DNA to distinguish these sets of strains.

The PFGE patterns of Erwinia strains from Japan appeared unrelated to Erwinia amylovora, whereas they were highly conserved for all European strains assayed (Zhang \& Geider, 1997; Zhang et al., 1998). A relationship to Erwinia amylovora strains isolated from Rubus sp. could also be excluded by additional molecular data obtained. Rubus sp. strains differ from fruit tree strains in their PFGE pattern (S. Jock \& K. Geider, unpublished), but they share an almost identical nucleotide sequence in a region upstream of the EPSsynthesis-encoding gene clusters and do not only produce PCR signals with pEA29 targeted primers, but also with ams primers used to identify strains from fruit trees and ornamentals. Similar to Erwinia pyrifoliae found in Korea, which have variable PFGE patterns, the Japanese erwinias appear to be also more variable than Erwinia amylovora. BIOLOG profiles and DNA hybridizations with the hrp region of Erwinia amylovora placed the Japanese strains close to the fire blight pathogen (Beer $e t$ al., 1996). Other criteria, such as molecular features, including the nucleotide sequences of a DNA fragment from a chromosomal region preceding the gene cluster for EPS synthesis, seem to be quite powerful for differentiation of the Japanese Erwinia strains from Erwinia amylovora, although they are not classical taxonomic criteria. After the molecular differentiation of the Erwinia strains from Japan, molecular criteria proposed for the detection and identification of fire blight such as specific PCR primers (Bereswill et al., 1992, 1995; Kim \& Geider, 1999) are still valid for diagnosis of the disease and were recently successfully applied to describe a temporary infection of plants with Erwinia amylovora in the Melbourne Botanic Gardens (Jock et al., 2000). Consequently, previous reports about fire blight in Japan on the island of Hokkaido (Beer et al., 1996; Kim et al., 1996) have to be reconsidered in the light of the new findings that the pear-pathogenic bacteria from Japan are more closely related to the Korean pear pathogen Erwinia pyrifoliae than to the fire blight pathogen Erwinia amylovora.

\section{ACKNOWLEDGEMENTS}

We thank Drs S. Beer and J. Kim (Cornell University, Ithaca, NY, USA) for providing strains listed in Table 1 and D. Coplin (Ohio State University, Columbus, OH, USA), A. Jones (Michigan State University, East Lansing, MI, USA) and S. Thomson (Utah State University, Logan, UT, USA) for comments on the manuscript.

\section{REFERENCES}

Beer, S. V., Kim, J.-H., Zumoff, C. H. \& 7 other authors (1996). Characterization of bacteria that cause 'bacterial shoot blight of pear' in Japan. Acta Hortic 411, 179-181.

Bereswill, S., Pahl, A., Bellemann, P., Zeller, W. \& Geider, K. (1992). Sensitive and species-specific detection of Erwinia amylovora by polymerase chain reaction analysis. Appl Environ Microbiol 58, 3522-3526.

Bereswill, S., Bugert, P., Bruchmüller, I. \& Geider, K. (1995). Identification of Erwinia amylovora by PCR with chromosomal DNA. Appl Environ Microbiol 61, 2636-2642.

Bereswill, S., Jock, S., Bellemann, P. \& Geider, K. (1998). Identification of Erwinia amylovora by growth morphology on 
agar containing copper sulfate and by capsule staining with lectin. Plant Dis 82, 158-164.

Bugert, P. \& Geider, K. (1995). Molecular analysis of the amsoperon required for exopolysaccharide synthesis of Erwinia amylovora. Mol Microbiol 15, 917-933.

Chung, Y. R., Brenner, D. J., Steigerwalt, A. G., Kim, B. S., Kim, H. T. \& Cho, K. Y. (1993). Enterobacter pyrinus sp. nov., an organism associated with brown leaf spot disease of pear trees. Int J Syst Bacteriol 43, 157-161.

Falkenstein, H., Bellemann, P., Walter, S., Zeller, W. \& Geider, K. (1988). Identification of Erwinia amylovora, the fireblight pathogen, by colony hybridization with DNA from plasmid pEA29. Appl Environ Microbiol 54, 2798-2802.

Goto, M. (1992). Fundamentals of Bacterial Plant Pathology. San Diego: Academic Press.

Hugh, R. \& Leifson, E. (1953). The taxonomic significance of fermentative versus oxidative metabolism of carbohydrates by various Gram-negative bacteria. J Bacteriol 66, 24.

Jock, S., Rodoni, B., Gillings, M., Kim, W.-S., Copes, C., Merriman, P. \& Geider, K. (2000). Screening of ornamental plants from the Botanic Gardens of Melbourne and Adelaide for the occurrence of Erwinia amylovora. Australas Plant Pathol 29, 120-128.

Kim, J.-H., Beer, S. V., Zumoff, C. H., Laby, R. J., Gustafson, H. L., Aldwinckle, H. S. \& Tanii, A. (1996). Characterization of Erwinia amylovora strains from different hosts and geographical areas. Acta Hortic 411, 183-186.

Kim, W.-S. \& Geider, K. (1999). Analysis of variable shortsequence DNA repeats on the $29 \mathrm{~kb}$ plasmid of Erwinia amylovora strains. Eur J Plant Pathol 108, 703-713.

Kim, W.-S., Gardan, L., Rhim, S.-L. \& Geider, K. (1999). Erwinia pyrifoliae sp. nov., a novel pathogen affecting Asian pear trees (Pyrus pyrifolia Nakai). Int J Syst Bacteriol 49, 899-906.
Kim, W.-S., Jock, S., Paulin, J.-P., Rhim, S.-L. \& Geider, K. (2001). Molecular detection and differentiation of Erwinia pyrifoliae and host range analysis of the Asian pear pathogen. Plant Dis $\mathbf{8 5}$ (in press).

McGhee, G. C. \& Jones, A. L. (2000). Complete nucleotide sequence of ubiquitous plasmid pEA29 from Erwinia amylovora strain Ea88: Gene organization and intraspecies variation. Appl Environ Microbiol 66, 4897-4907.

Nei, M. \& Li, W.-H. (1979). Mathematical model for studying genetic variation in terms of restriction nucleases. Proc Natl Acad Sci US A 76, 5269-5273.

Rhim, S.-L., Völksch, B., Gardan, L., Paulin, J.-P., Langlotz, C., Kim, W.-S. \& Geider, K. (1999). Erwinia pyrifoliae, an Erwinia species, different from Erwinia amylovora, causes a necrotic disease of Asian pear trees. Plant Pathol 48, 514-520.

Sambrook, J., Fritsch, E. F. \& Maniatis, T. (1989). Molecular Cloning: a Laboratory Manual, 2nd edn. Cold Spring Harbor, NY: Cold Spring Harbor Laboratory.

Weisburg, W. G., Barns, S. M., Pelletier, D. A. \& Lane, D. J. (1991). 16 S ribosomal DNA amplification for phylogenetic study. $J$ Bacteriol 173, 697-703.

Zhang, Y. \& Geider, K. (1997). Differentiation of Erwinia amylovora strains by pulsed-field gel electrophoresis. Appl Environ Microbiol 63, 4421-4426.

Zhang, Y., Merighi, M., Bazzi, C. \& Geider, K. (1998). Genomic analysis by pulsed-field gel electrophoresis of Erwinia amylovora strains from the Mediterranean region including Italy. J Plant Pathol 80, 225-232.

Received 22 January 2001; revised 27 June 2001; accepted 2 July 2001. 